IRA-International Journal of Applied Sciences ISSN 2455-4499; Vol.07, Issue 02 (2017)

Institute of Research Advances

Pg. no. 52-61

http://research-advances.org/index.php/IRAJAS

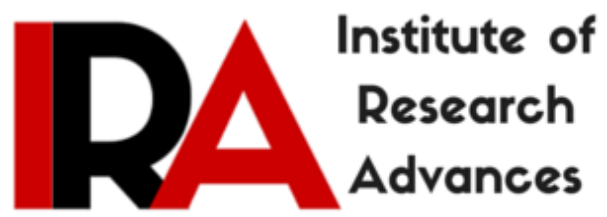

\title{
Underground Water Quality in Major Paddy Plants Growing Area of Mayiladuthurai Taluk of Nagapattinam District in Tamil Nadu - India
}

\author{
${ }^{1}$ A.Vincentraj, ${ }^{2}$ S.Kalyanasundharam, ${ }^{3}$ A.Arokiyaraj, ${ }^{4}$ N. Pasupathy, ${ }^{5}$ S. Leo \\ Arokiaraj and ${ }^{6}$ D.Sathya \\ ${ }^{1 \& 2}$ PG and Research Department of Chemistry, Poompuhar College (Autonomous), \\ Melaiyur-609 107, Nagapattinam, Tamilnadu, India. \\ ${ }^{3 \& 4}$ PG and Research Department of Chemistry, A.V.C.College (Autonomous), \\ Mannampandal-609 305, Nagapattinam, Tamilnadu, India. \\ 5\&6 Department of Chemistry, Kalaimahal College of Arts and Science, Sembanar koil- \\ 609 309, Nagapattinam, Tamilnadu, India.
}

Type of Review: Peer Reviewed.

DOI: http://dx.doi.org/10.21013/jas.v7.n2.p1

\section{How to cite this paper:}

Vincentraj, A., Kalyanasundharam, S., Arokiyaraj, A., Pasupathy, N., Arokiaraj, S., \& Sathya, D. (2017). Underground Water Quality in Major Paddy Plants Growing Area of Mayiladuthurai Taluk of Nagapattinam District in Tamil Nadu - India. IRA-International Journal of Applied Sciences (ISSN 2455-4499), 7(2), 52-61. doi:http://dx.doi.org/10.21013/jas.v7.n2.p1

(C) Institute of Research Advances

\section{(cc) EY-NC}

This work is licensed under a Creative Commons Attribution-Non Commercial 4.0 International License subject to proper citation to the publication source of the work.

Disclaimer: The scholarly papers as reviewed and published by the Institute of Research Advances (IRA) are the views and opinions of their respective authors and are not the views or opinions of the IRA. The IRA disclaims of any harm or loss caused due to the published content to any party. 
Water is essential for agriculture. Most of the farmers depending upon the ground water for irrigation purpose, but now a days the underground water is going to towards very deep down level. Because our environment affected by water drought, pollution and lack of rain falls. It will be creating negative effects to cultivation. In this present study is to assess the underground water quality in Mayiladuthurai Taluk of Nagapattinam District in Tamil Nadu. Totally 30 underground water samples were collected, which covers 10 Revenue villages in Mayiladuthurai Taluk, 3 samples from each Revenue village and analyzed all the physico - chemical parameters such as $\mathrm{pH}, \mathrm{EC}, \mathrm{Ca}, \mathrm{Na}, \mathrm{K}, \mathrm{SO}_{4}, \mathrm{Cl}, \mathrm{Mg}, \mathrm{CO}_{3}$, and $\mathrm{HCO}_{3}$ and the water quality index SAR, RSC, Geo-chemical types, classification were determined by standard methods, standard calculation and by using standard instruments. Then the irrigation water quality results were compared with standard values Recommended by World Health Organization (WHO), Bureau of Indian Standards (BIS) and Indian Council of Medical Research (ICMR). The proposed work is very essential not only for crop production but also to maintain soil fertility, to maintain hazardous free environment and to enhance the living standard and in turn to uplift our Agriculturist.

Keywords: Irrigation Water, Mayiladuthurai Taluk, Nagapattinam District, Tamil Nadu

\section{Introduction}

Ground water plays an important vital in agriculture, for both watering of crops and for irrigation of dry season crops. It is estimated that about $45 \%$ of irrigation water requirement is met from ground water sources. The quality of ground water is changing from place to place along with the depth. It also varies with seasonal changes and is primarily governed by the extent and composition of dissolved solids present in it. In most of the instances, the extraction of excessive quantities of ground water has resulted in drying up of wells, damaged ecosystems, land subsidence, salt-water intrusion and depletion of the resource. The concentration and composition of dissolved salts in water decides its suitability for irrigation, degradation of ground water quality of the aquifers causing sea water interference into the coastal aquifers ${ }^{[1]}$.Water samples analyzed by standard methods ${ }^{[2-6]}$. Total concentrations of soluble salts, relative quantity of sodium to other cations and the bicarbonate concentration related to the concentration of calcium plus magnesium are important characteristics determining the quality of irrigation water. Salinity of irrigation water may limit the choice of crops that can be grown. The most influential water quality guideline on crop productivity is the water salinity hazard as measured by electrical conductivity. ${ }^{[7]}$

\section{Study Area}

The study area is Mayiladuthurai Taluk of Nagapattinam district coastal region in the southern Tamilnadu State located in the coastal region of Bay of Bengal 11.0290373 Latitude and 79.8506815 Longitude. This taluk is spread over in 27,726 hectares of Agriculture land. Fig.1 shows the study area.

\section{Materials and Methods}

Irrigation water samples were collected randomly in a systematic manner covering major rice growing area in Mayiladuthurai taluk of Nagapattinam district. A total 30 samples were collected from directly bore wells in clean plastic cans of 1 liter capacity. The bottles are tagged individually for sufficient information similar to date, location, deepness of well. The results of the average mean value of irrigation water quality parameters are shown in Table.10. The percentage of irrigation water quality parameters are shown in Table.11. Quality of irrigation water analyzed in the laboratory determined many parameters such as $\mathrm{pH}, \mathrm{EC}, \mathrm{Ca}, \mathrm{Na}, \mathrm{K}, \mathrm{SO}_{4}, \mathrm{Cl}, \mathrm{Mg}, \mathrm{CO}_{3}$, and $\mathrm{HCO}_{3}$ are determined by standard methods and by using standard instruments. Then the irrigation water quality results are compared with standard values Recommended by World Health Organization (WHO), Bureau of Indian Standards (BIS) and Indian Council of Medical Research (ICMR). 


\section{Important water quality ratings}

Important water quality ratings that merit consideration are detailed below, from table (1 to 8). These ratings could be used as guidelines when recommendations are given.

\section{Sodium Absorption Ratio (SAR)}

The ratio of sodium contents to that of calcium and magnesium in the water is called Sodium Absorption Ratio. Sodium Absorption Ratio also denotes sodium hazard (table.7).

$\mathrm{SAR}=\mathrm{Na}^{2+} / \sqrt{\mathrm{Ca}^{2+}} \mathrm{Mg}^{2+} / 2$

\section{Residual Sodium Carbonate (RSC)}

Residual Sodium Carbonate indicates bicarbonate hazard. It is determined by the proportion of carbonate ions to that of calcium and magnesium ions (table.8)

$\operatorname{RSC}(\mathrm{Meq} / \mathrm{lit})=\left(\mathrm{CO}_{3}{ }^{2+}+\mathrm{HCO}_{3}{ }^{2+)}-\left(\mathrm{Ca}^{2+}+\mathrm{Mg}^{2+}\right)\right.$

The increased Residual Sodium Carbonate value leads to alkali formation because of the precipitation of calcium and magnesium carbonate/ bicarbonate or sodium and bicarbonate

\section{Geo Chemical Type}

Calcium bicarbonate and calcium sulphate waters contain the lowest conductance. Sodium chloride water has the highest conductance. When calcium and magnesium more than half of the total cations it is considered calcium/ magnesium type ${ }^{[7]}$. When sodium and potassium is more than half of the total cations, the water is considered sodium type, if bicarbonate, carbonate is than $50 \%$ of the total anions, it is considered bicarbonate type. The content of chloride plus sulphate exceeds $50 \%$ of the total anions it is considered chloride type. Problems associated with some Geo chemical types of water. Geo chemical type refers to predominate soluble salt present in the irrigation water. The wide Geo chemical types are chloride water, sulphate water, bicarbonate water. Associating with different cations, the Geo chemical type varies. Magnesium content is the important in determining the quality of irrigation water, sodium is another essential factor to study sodium hazard. The high percentage of sodium water stunts the plant growth, sodium react with soil to decrease its permeability ${ }^{[8]}$.Sodium percent in water is a parameter computed to evaluate the suitability for irrigation ${ }^{[9]}$.Excess sodium water develop the unwanted effects of changing soil properties and reducing soil permeability ${ }^{[10]}$, the irrigation water tends to enter into cation-exchange reactions in soil can be indicated by the sodium absorption ratio ${ }^{[11]}$.The irrigation water status of mayiladuthurai taluk were given figure 2 to 6 .

\section{Result and Discussion}

Assess the 30 irrigation water samples collected from 10 revenue villages in Mayiladuthurai taluk by collecting 3 water samples per village revealed that water analysis parameter in Mayiladuthurai Taluk, water samples with mean status of the $C_{1} S_{1} R_{1}$ classification (table.10 and fig.4 to 6$)$.

The irrigation water status of Mayiladuthurai taluk is given in Table 10 to11 and, fig.2 to 6. Based on the electrical conductivity classification, $53.3 \%$ of villages covers under $\mathrm{C}_{1}$ classification, $30 \%$ of villages covers under $\mathrm{C}_{2}$ classification, and $\mathrm{C}_{3}$ classification is $16.7 \%$. In Mayiladuthurai taluk, $53.3 \%$ of villages cover under $\mathrm{C}_{1}$ classification due to basin area. Based on Sodium Absorption Ratio classification, $100 \%$ of villages cover under $\mathrm{S}_{1}$ classification. $\mathrm{S}_{2}$ and $\mathrm{S}_{3}$ classifications are not found. In $100 \%$ villages covers $S_{1}$ classification due to non-saline water. Based on Residual Sodium Carbonate classification, $96.6 \%$ villages cover under $R_{1}$ classification, $3.3 \%$ for $R_{2}$ and $R_{3}$ are not found. 


\section{Conclusion}

The present study assesses the water quality status of Mayiladuthurai taluk exposed that they have become a significant trouble (Yield limiting factor) in Mayiladuthurai taluk. Hence the suggestion for different type of water for better yield. Sodium and chloride such water could be improved by gypsum application. Chemical amendments are aimed at to introduce favorable cationic ratios. If water contains high sodium and magnesium bicarbonates, gypsum can be added to irrigation water. Soils irrigated with poor quality water are low in fertility particularly nitrogen. Nitrogen response in good when is applied along with manure, under saline and alkaline soils, should be avoided due to nitrogen loss.

\section{References}

1. B. Chandrasekaran, S Anbazhagan (2007),Variability of soil-water quality due to Tsunami2004 in the coastal belt of Nagapattinam district, Tamilnadu.

2. APHA standard methods for examination of water and waste,American Public Health Association,Washington D.C.(1998).

3. Manual on water and waste water analysis,NEERI Publications(1998).

4. D.R Khanna. Ecology and pollution of Ganga River,Ashish Publishing House,Delhi,1(1993).

5. Korfali and jurdi.Int.J.Environmental and pollution,19,271(2003).

6. Manual on water and waste water analysis,NEERI Publications(1998).

7. S.S. Ahmed, Q.H. Mazumder, C. S.Jahan, M.Ahmed and S.Islam. Journal, Geological society of India, 60, 411 (2002).

8. D.K Todd., Groundwater Hydrology, 2nd Ed. John Wiley and Sons, New York .p-535. (1980).

9. P.C. Mishra. Thesis- Some aspects of water quality of water in and around Rourkela. Department of Chemistry, National Institute of Technology, Rourkela (2005).

10. S.N. Biswas, H. Mohabey and M.L. Malik. Asian J. Chem., 16, (2002).

11. V.Sing and C.C.P. Singh. Asian J. Chem., 18, 2813 (2004). Table-1 : Irrigation water characteristics in winter season. 
(Tables \& Figures)

Table. 1 Water Quality Based on EC,SAR,RSC

\begin{tabular}{llll}
\hline \multicolumn{1}{c}{ Water Quality } & \multicolumn{1}{c}{ EC $\left(\mathrm{dsm}^{-1}\right)$} & \multicolumn{1}{c}{$\begin{array}{c}\text { SAR } \\
(\mathrm{Meq} / \mathrm{lit})\end{array}$} & \multicolumn{1}{c}{$\begin{array}{c}\text { RSC } \\
\text { (Meq/lit) }\end{array}$} \\
\hline Good & Less than 2.0 & Less than 10.0 & Nil \\
Marginal water & $2-4$ & Less than 10.0 & Less than 2.5 \\
Saline water & More than 4.0 & Less than 10.0 & Nil \\
Sodic water & Less than 4.0 & More than 10.0 & Usually more than 2.5 \\
\hline
\end{tabular}

Table. 2 Water Quality Based on Sodium

\begin{tabular}{ll}
\hline Low sodium water & Could be used in almost all soils. \\
\hline Medium.sodium water & $\begin{array}{l}\text { Considerable sodium hazard in high clay soils and low organic } \\
\text { matter under low discharge conditions unless gypsum is present } \\
\text { in the soils. }\end{array}$ \\
High sodium water & $\begin{array}{l}\text { Sodium hazard is significant in non-gypsiferous soils. Good } \\
\text { drainage, good discharge and organic matter are wanted. }\end{array}$ \\
$\begin{array}{l}\text { Chemical amendments may be used for transferable sodium } \\
\text { replacement expect for high salinity water. }\end{array}$
\end{tabular}

Table. 3 Water Quality Based on Electrical Conductivity

\begin{tabular}{|c|c|c|}
\hline Low salinity water & $0-250\left(\mathrm{dsm}^{-1}\right)$ & $\begin{array}{l}\text { Used for most crops on most Soils with little } \\
\text { possibility of Salinity some leaching is } \\
\text { required. }\end{array}$ \\
\hline Moderate salinity water & $250-750\left(\mathrm{dsm}^{-1}\right)$ & $\begin{array}{l}\text { All the crops except extremely salt sensitive } \\
\text { crops, when grown on soils of high to medium } \\
\text { permeability. Leaching precaution and } \\
\text { moderate salt tolerant crops. }\end{array}$ \\
\hline Medium to high salinity & $750-2250\left(\mathrm{dsm}^{-1}\right)$ & $\begin{array}{l}\text { Should be used only on soils of moderate to } \\
\text { good permeability. Regular leaching and plants } \\
\text { with moderate to good tolerance to salinity. }\end{array}$ \\
\hline High salinity water & $2250-6000$ & $\begin{array}{l}\text { To be used only on soils of good permeability } \\
\text { with regular leaching. Plants with high } \\
\text { tolerance. }\end{array}$ \\
\hline Excess salinity water & More than $6000\left(\mathrm{dsm}^{-1}\right)$ & Should not be used. \\
\hline
\end{tabular}


Table. 4\&5 Salinity classification

\begin{tabular}{llll}
\hline Low to moderate salinity & $0-750$ & $\mathrm{NaCl}-1$ & $\mathrm{MgCl}_{2}-1$ \\
\hline Medium to high salinity & $750-2250$ & $\mathrm{NaCl}-2$ & $\mathrm{MgCl}_{2}-2$ \\
High salinity & $2250-4000$ & $\mathrm{NaCl}-3$ & $\mathrm{MgCl}_{2}-3$ \\
Very high salinity & $4000-6000$ and above & $\mathrm{NaCl}-4$ & $\mathrm{MgCl}_{2}-4$ \\
\hline \multicolumn{2}{l}{} \\
\hline Low to medium & $0-2250$ & $\mathrm{CaCl}_{2-1}$ & \\
\hline High & $2250-4000$ & $\mathrm{CaCl}_{2}-2$ & \\
Very high & $4000-6000$ & $\mathrm{CaCl}_{2-3}$ & \\
Excessive & More than 6000 & $\mathrm{CaCl}_{2-4}-4$ & \\
\hline
\end{tabular}

Table. 6 Classification of irrigation water based on Electrical Conductivity

\begin{tabular}{llll}
\hline Class & E.C $\left(\mathrm{dsm}^{-1}\right)$ & Salinity status & Suitability \\
\hline $\mathrm{C}_{1}$ & $0.0-1.0$ & Low salinity water & Excellent \\
$\mathrm{C}_{2}$ & $1.01-2.0$ & Medium salinity water & Good \\
$\mathrm{C}_{3}$ & $2.01-4.0$ & Salinity water & Doubtful \\
$\mathrm{C}_{4}$ & $4.01-6.0$ & High salinity water & Injurious \\
$\mathrm{C}_{5}$ & $>6.0$ & Very high salinity water & Unsuitable \\
\hline
\end{tabular}

Table.7 Classification of irrigation water based on Sodium Absorption Ratio

\begin{tabular}{lll}
\hline Class & SAR & Suitability \\
\hline S1 & $<10$ & Safe \\
S2 & $10.01-20.0$ & Moderate \\
S3 & $>20.0$ & Unsafe \\
\hline
\end{tabular}

Table. 8 Classification of irrigation water based on Residual Sodium Carbonate

\begin{tabular}{lll}
\hline Class & RSC $($ Meq/lit $)$ & Suitability \\
\hline R1 & $<1.25$ & Safe \\
R2 & $1.26-2.50$ & Moderate \\
R3 & $>2.50$ & Unsafe \\
\hline
\end{tabular}


Table No. 9- Irrigation Water Quality Parameters of Mayiladuthurai Taluk of Nagapattinam District.

\begin{tabular}{|c|c|c|c|c|c|c|c|c|c|c|c|c|c|c|c|c|c|c|}
\hline \multirow[t]{2}{*}{ No } & \multirow{2}{*}{$\begin{array}{l}\mathscr{\Xi} \\
\stackrel{\infty}{\Xi} \\
\stackrel{\Xi}{\Xi}\end{array}$} & \multirow{2}{*}{ 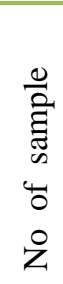 } & \multirow[b]{2}{*}{$\frac{\pi}{2}$} & \multirow{2}{*}{ 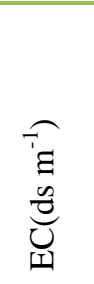 } & \multicolumn{2}{|c|}{$\begin{array}{l}\text { ANIONS } \\
\text { (meq/lit) }\end{array}$} & \multirow[b]{2}{*}{$\bar{U}$} & \multirow[b]{2}{*}{$\bigcup_{\mathscr{S}}^{+}$} & \multirow[b]{2}{*}{ 푱 } & \multicolumn{5}{|c|}{$\begin{array}{l}\text { CATIONS } \\
\text { (meq/lit) }\end{array}$} & \multirow[b]{2}{*}{ 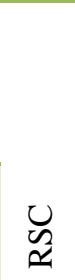 } & \multirow[b]{2}{*}{$\underset{c}{\stackrel{\alpha}{c}}$} & \multirow[t]{2}{*}{ Type } & \multirow[t]{2}{*}{ Class } \\
\hline & & & & & రీ & 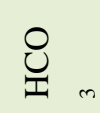 & & & & $\tilde{U}$ & $\sum^{\infty}$ & $\tilde{z}$ & 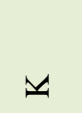 & ]ٓ & & & & \\
\hline \multirow[t]{3}{*}{1} & Maharajapuram & 3 & 7.9 & 0.66 & 0.5 & 3.6 & 2.5 & - & 15.16 & 2.2 & 1.3 & 3.0 & 0.14 & 6.64 & 0.6 & 2.3 & $\mathrm{NaHCO}_{3}$ & $\mathrm{C}_{1} \mathrm{~S}_{1} \mathrm{R}_{1}$ \\
\hline & & & 8.0 & 0.69 & 0.6 & 3.4 & 2.9 & - & 15.59 & 1.8 & 1.7 & 3.2 & 0.19 & 6.89 & 0.5 & 2.4 & $\mathrm{NaHCO}_{3}$ & $\mathrm{C}_{1} \mathrm{~S}_{1} \mathrm{R}_{1}$ \\
\hline & & & 8.1 & 1.26 & 0.4 & 7.9 & 4.3 & - & 21.96 & 2.8 & 3.2 & 6.5 & 0.18 & 12.68 & 2.3 & 3.75 & $\mathrm{NaHCO}_{3}$ & $\mathrm{C}_{2} \mathrm{~S}_{1} \mathrm{R}_{1}$ \\
\hline \multirow[t]{3}{*}{2} & Ponnur & 3 & 7.6 & 2.5 & - & 5.7 & 19.3 & - & 35.1 & 6.1 & 8.2 & 10.39 & 0.27 & 24.96 & - & 3.89 & $\mathrm{NaCl}$ & $\mathrm{C}_{3} \mathrm{~S}_{1} \mathrm{R}_{1}$ \\
\hline & & & 7.8 & 2.8 & 0.2 & 5.7 & 22.1 & - & 38.6 & 7.4 & 9.1 & 11.24 & 0.29 & 28.03 & - & 3.91 & $\mathrm{NaCl}$ & $\mathrm{C}_{3} \mathrm{~S}_{1} \mathrm{R}_{1}$ \\
\hline & & & 7.9 & 1.6 & 0.2 & 7.5 & 8.3 & - & 25.5 & 4.9 & 3.7 & 7.15 & 0.23 & 15.98 & - & 3.45 & $\mathrm{NaCl}$ & $\mathrm{C}_{2} \mathrm{~S}_{1} \mathrm{R}_{1}$ \\
\hline \multirow[t]{3}{*}{3} & Pondur & 3 & 7.9 & 0.86 & 0.2 & 6.2 & 0.2 & - & 15.36 & 1.5 & 3.7 & 3.24 & 0.2 & 8.64 & 1.2 & 2.01 & $\mathrm{Mg} \mathrm{HCO}_{3}$ & $\mathrm{C}_{1} \mathrm{~S}_{1} \mathrm{R}_{1}$ \\
\hline & & & 7.2 & 2.5 & - & 11.2 & 13.6 & 0.2 & 34.7 & 6.7 & 11.9 & 6.27 & 0.16 & 25.03 & - & 2.06 & $\mathrm{MgCl}_{2}$ & $\mathrm{C}_{3} \mathrm{~S}_{1} \mathrm{R}_{1}$ \\
\hline & & & 8.0 & 0.72 & 0.2 & 4.2 & 2.8 & - & 15.92 & 2.0 & 2.1 & 2.9 & 0.2 & 7.2 & 0.3 & 0.49 & $\mathrm{NaHCO}_{3}$ & $\mathrm{C}_{1} \mathrm{~S}_{1} \mathrm{R}_{1}$ \\
\hline \multirow[t]{3}{*}{4} & Thirumanglam & 3 & 8.1 & 0.69 & 0.2 & 4.0 & 2.7 & - & 15.69 & 1.4 & 2.4 & 2.91 & 0.19 & 6.9 & 0.4 & 0.47 & $\mathrm{NaHCO}_{3}$ & $\mathrm{C}_{1} \mathrm{~S}_{1} \mathrm{R}_{1}$ \\
\hline & & & 7.7 & 0.61 & 0.2 & 3.1 & 2.8 & - & 14.41 & 1.4 & 1.9 & 2.61 & 0.19 & 6.1 & - & 0.49 & $\mathrm{NaHCO}_{3}$ & $\mathrm{C}_{1} \mathrm{~S}_{1} \mathrm{R}_{1}$ \\
\hline & & & 8.0 & 1.38 & 1.0 & 5.2 & 7.6 & - & 23.18 & 3.7 & 2.9 & 6.9 & 0.30 & 13.8 & - & 0.26 & $\mathrm{NaCl}$ & $\mathrm{C}_{2} \mathrm{~S}_{1} \mathrm{R}_{1}$ \\
\hline \multirow[t]{3}{*}{5} & Kali & 3 & 6.8 & 0.25 & - & 1.7 & 0.8 & - & 9.55 & 1.4 & 0.8 & 0.17 & 0.15 & 2.52 & - & 0.16 & $\mathrm{Ca} \mathrm{HCO}_{3}$ & $\mathrm{C}_{1} \mathrm{~S}_{1} \mathrm{R}_{1}$ \\
\hline & & & 7.4 & 0.66 & 0.2 & 4.1 & 2.3 & - & 14.66 & 2.1 & 1.5 & 2.77 & 0.19 & 6.56 & 0.7 & 2.01 & $\mathrm{NaHCO}_{3}$ & $\mathrm{C}_{1} \mathrm{~S}_{1} \mathrm{R}_{1}$ \\
\hline & & & 6.7 & 0.74 & - & 2.3 & 5.1 & - & 14.84 & 2.7 & 1.5 & 3.0 & 0.2 & 7.4 & - & 2.07 & $\mathrm{NaCl}$ & $\mathrm{C}_{1} \mathrm{~S}_{1} \mathrm{R}_{1}$ \\
\hline \multirow[t]{3}{*}{6} & Kadalangudi & 3 & 7.0 & 0.83 & 0.4 & 4.1 & 3.8 & - & 16.13 & 3.2 & 1.9 & 3.1 & 0.1 & 8.3 & - & 1.94 & $\mathrm{Ca} \mathrm{HCO}_{3}$ & $\mathrm{C}_{1} \mathrm{~S}_{1} \mathrm{R}_{1}$ \\
\hline & & & 7.0 & 0.53 & - & 2.6 & 2.7 & - & 12.83 & 1.2 & 2.8 & 1.2 & 0.1 & 5.3 & - & 0.85 & $\mathrm{MgCl}_{2}$ & $\mathrm{C}_{1} \mathrm{~S}_{1} \mathrm{R}_{1}$ \\
\hline & & & 5.9 & 0.99 & - & 5.2 & 4.7 & - & 16.79 & 3.2 & 4.1 & 2.5 & 0.16 & 9.96 & - & 1.31 & $\mathrm{Mg} \mathrm{HCO}_{3}$ & $\mathrm{C}_{1} \mathrm{~S}_{1} \mathrm{R}_{1}$ \\
\hline \multirow[t]{3}{*}{7} & Athur & 3 & 7.1 & 0.87 & - & 3.7 & 5.0 & - & 16.67 & 2.9 & 2.5 & 3.1 & 0.17 & 8.67 & - & 1.89 & $\mathrm{NaCl}$ & $\mathrm{C}_{1} \mathrm{~S}_{1} \mathrm{R}_{1}$ \\
\hline & & & 7.4 & 1.32 & 0.2 & 6.9 & 5.9 & 0.2 & 21.92 & 3.8 & 4.5 & 4.69 & 0.21 & 13.2 & - & 2.31 & $\mathrm{NaHCO}_{3}$ & $\mathrm{C}_{2} \mathrm{~S}_{1} \mathrm{R}_{1}$ \\
\hline & & & 7.1 & 1.60 & - & 8.7 & 7.1 & 0.2 & 24.7 & 3.6 & 4.6 & 7.57 & 0.16 & 15.93 & - & 3.74 & $\mathrm{NaHCO}_{3}$ & $\mathrm{C}_{2} \mathrm{~S}_{1} \mathrm{R}_{1}$ \\
\hline \multirow[t]{3}{*}{8} & Manalmedu & 3 & 7.5 & 1.93 & - & 6.4 & 12.7 & 0.2 & 28.73 & 6.5 & 6.6 & 6.03 & 0.16 & 19.29 & - & 2.36 & $\mathrm{MgCl}_{2}$ & $\mathrm{C}_{2} \mathrm{~S}_{1} \mathrm{R}_{1}$ \\
\hline & & & 7.4 & 1.82 & - & 6.6 & 11.4 & 0.2 & 27.42 & 5.8 & 7.0 & 5.14 & 0.31 & 18.25 & - & 2.04 & $\mathrm{MgCl}_{2}$ & $\mathrm{C}_{2} \mathrm{~S}_{1} \mathrm{R}_{1}$ \\
\hline & & & 7.2 & 0.76 & - & 5.4 & 2.2 & - & 15.56 & 2.1 & 3.0 & 2.34 & 0.23 & 7.67 & 0.3 & 1.47 & $\mathrm{Mg} \mathrm{HCO}_{3}$ & $\mathrm{C}_{1} \mathrm{~S}_{1} \mathrm{R}_{1}$ \\
\hline \multirow[t]{3}{*}{9} & Pattavarthi & 3 & 8.0 & 1.08 & 0.7 & 6.6 & 3.5 & - & 19.88 & 2.2 & 2.6 & 5.8 & 0.2 & 10.8 & 2.5 & 3.74 & $\mathrm{NaHCO}_{3}$ & $\mathrm{C}_{2} \mathrm{~S}_{1} \mathrm{R}_{2}$ \\
\hline & & & 6.5 & 0.82 & - & 4.8 & 3.4 & - & 15.52 & 3.0 & 1.5 & 3.56 & 0.18 & 8.24 & 0.3 & 2.37 & $\mathrm{NaHCO}_{3}$ & $\mathrm{C}_{1} \mathrm{~S}_{1} \mathrm{R}_{1}$ \\
\hline & & & 7.3 & 0.63 & - & 4.1 & 2.2 & - & 14.23 & 1.8 & 2.0 & 2.29 & 0.16 & 6.25 & 0.3 & 1.67 & $\mathrm{NaHCO}_{3}$ & $\mathrm{C}_{1} \mathrm{~S}_{1} \mathrm{R}_{1}$ \\
\hline \multirow[t]{3}{*}{10} & Thalainayur & 3 & 7.2 & 2.3 & - & 7.2 & 15.6 & 0.2 & 32.5 & 7.5 & 7.2 & 8.0 & 0.29 & 22.99 & - & 2.95 & $\mathrm{NaCl}$ & $\mathrm{C}_{3} \mathrm{~S}_{1} \mathrm{R}_{1}$ \\
\hline & & & 7.3 & 2.6 & - & 7.6 & 18.2 & 0.3 & 36 & 7.8 & 9.1 & 8.87 & 0.27 & 26.04 & - & 3.05 & $\mathrm{MgCl}_{2}$ & $\mathrm{C}_{3} \mathrm{~S}_{1} \mathrm{R}_{1}$ \\
\hline & & & 7.3 & 1.45 & - & 8.4 & 6.1 & - & 23.25 & 3.0 & 6.6 & 4.72 & 0.21 & 14.53 & - & 2.16 & $\mathrm{Mg} \mathrm{HCO}_{3}$ & $\mathrm{C}_{2} \mathrm{~S}_{1} \mathrm{R}_{1}$ \\
\hline
\end{tabular}


Table.10 Averagemean value of irrigation water quality parameters of mayiladuthurai taluk in nagapattinam district.

\begin{tabular}{|c|c|c|c|c|c|c|c|c|c|c|c|c|c|c|c|c|c|}
\hline \multirow[t]{2}{*}{$\begin{array}{l}\text { Sl.N } \\
\text { o }\end{array}$} & \multirow[b]{2}{*}{$\begin{array}{l}\text { NAME OF } \\
\text { THE TALUK }\end{array}$} & \multirow{2}{*}{$\stackrel{T}{2}$} & \multirow{2}{*}{ 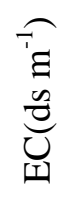 } & \multicolumn{5}{|c|}{$\begin{array}{c}\text { ANIONS } \\
\text { (meq/lit) }\end{array}$} & \multicolumn{5}{|c|}{$\begin{array}{c}\text { CATIONS } \\
\text { (meq/lit) }\end{array}$} & \multirow{2}{*}{$\begin{array}{l}U \\
\tilde{N}\end{array}$} & \multirow{2}{*}{ 䍃 } & \multirow[t]{2}{*}{$\begin{array}{c}\text { Typ } \\
\text { e }\end{array}$} & \multirow[t]{2}{*}{ Class } \\
\hline & & & & లో & $e_{0}^{0}$ & $\bar{U}$ & $0^{+}$ & 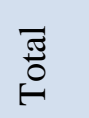 & $\tilde{U}$ & $\sum^{\infty}$ & $\ddot{z}$ & $\forall$ & స్ㅠㅇ & & & & \\
\hline 1 & $\begin{array}{l}\text { MAYILADU } \\
\text { THURAI } \\
\text { TALUK }\end{array}$ & $\begin{array}{c}7.4 \\
1\end{array}$ & $\begin{array}{c}1.2 \\
4\end{array}$ & $\begin{array}{l}0 . \\
3\end{array}$ & $\begin{array}{c}5.4 \\
7\end{array}$ & $\begin{array}{c}6.7 \\
2\end{array}$ & $\begin{array}{l}0 . \\
2\end{array}$ & $\begin{array}{c}21.0 \\
7\end{array}$ & 3.5 & 4.0 & $\begin{array}{c}4.7 \\
0\end{array}$ & $\begin{array}{c}0.1 \\
9\end{array}$ & $\begin{array}{c}12.4 \\
9\end{array}$ & $\begin{array}{l}0 . \\
8\end{array}$ & $\begin{array}{c}2.1 \\
1\end{array}$ & $\begin{array}{l}\mathrm{NaC} \\
1\end{array}$ & $\mathrm{C}_{1} \mathrm{~S}_{1} \mathrm{R}_{1}$ \\
\hline
\end{tabular}

Table.11 Percentage of Irrigation Water Quality Parameters of Mayiladuthurai Taluk in Nagapattinam District.

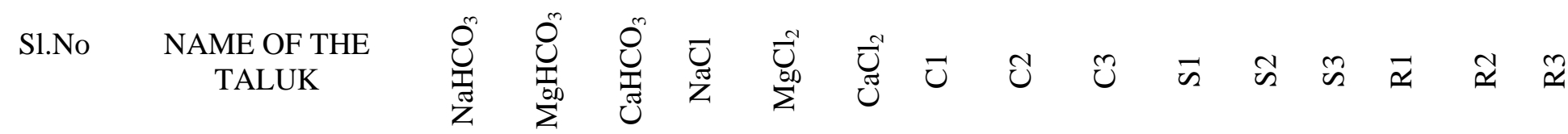

$\begin{array}{lllllllllllllll}\text { MAYILADUTHURAI } & 40.0 & 13.3 & 6.6 & 23.3 & 16.6 & - & 53.3 & 30.0 & 16.7 & 100 & - & - & 96.6 & 3.3\end{array}$ TALUK 
Fig.1. Location Map

Mayiladuthurai Taluk in Nagapattinam District
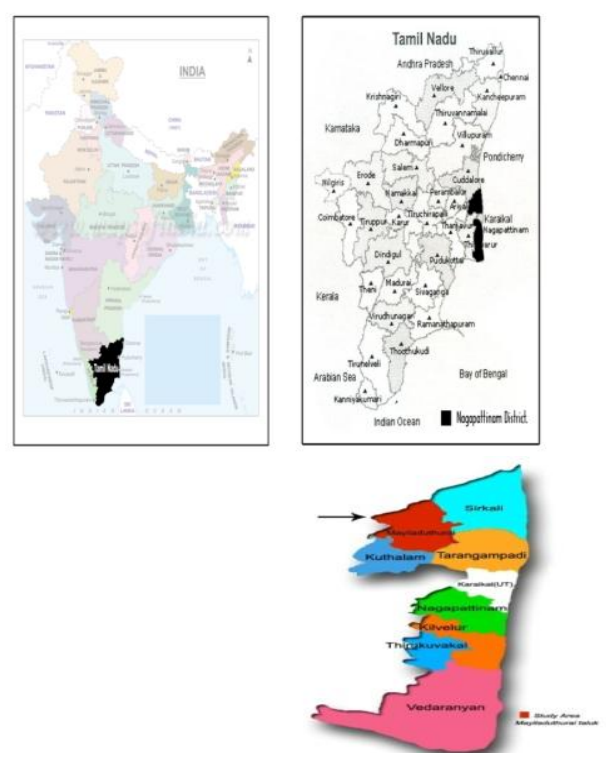

Figure .2 pH Status of Ten Revenue Villages of Mayiladuthurai Taluk

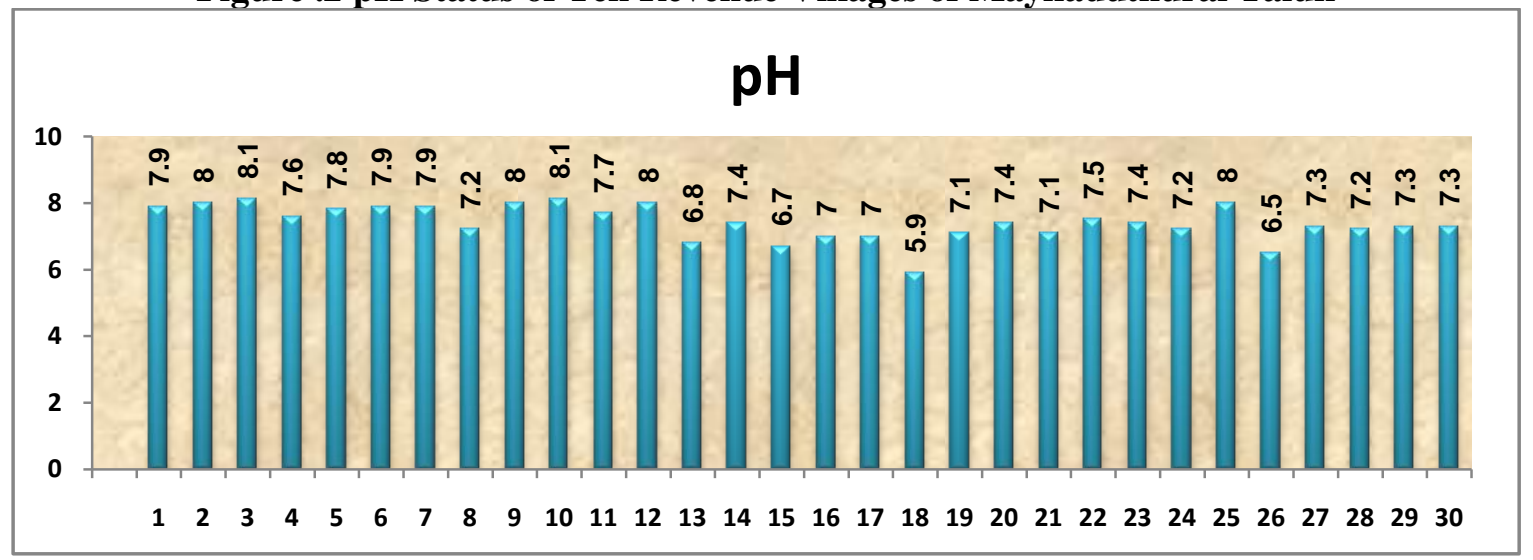

Figure. 3 EC Status of Ten Revenue Villages of Mayiladuthurai Taluk

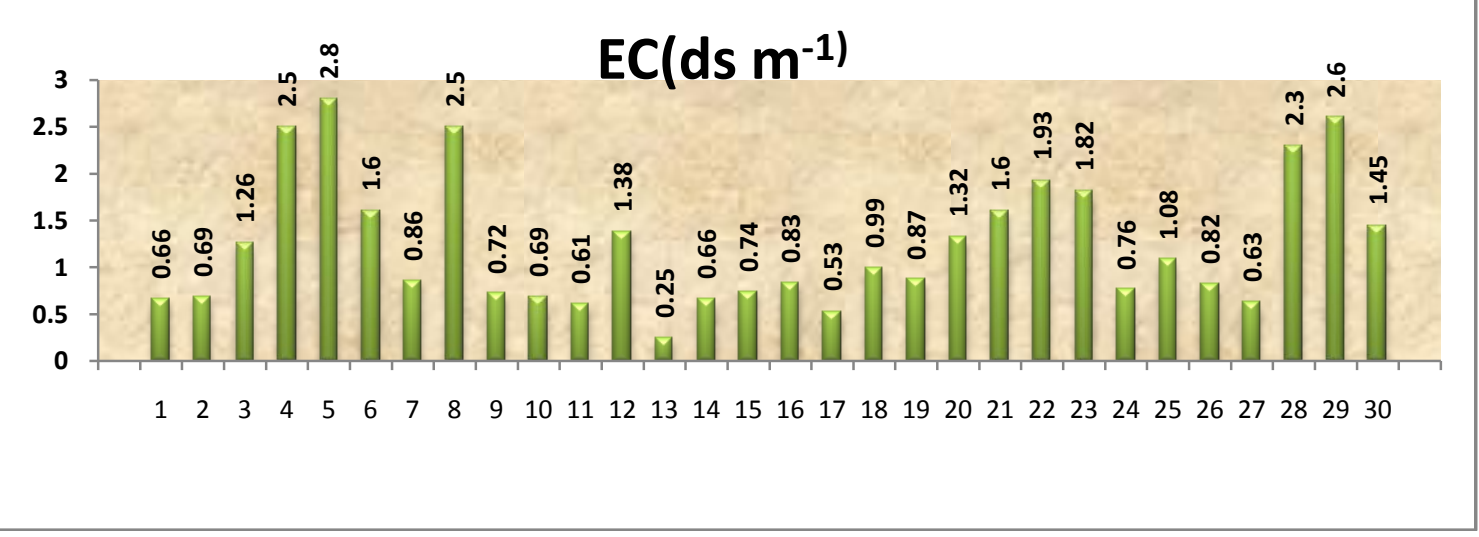




\section{IRRIGATION WATER STATUS OF MAYILADUTHURAI TALUK}

Figure. 4 Electrical Conductivity Rating

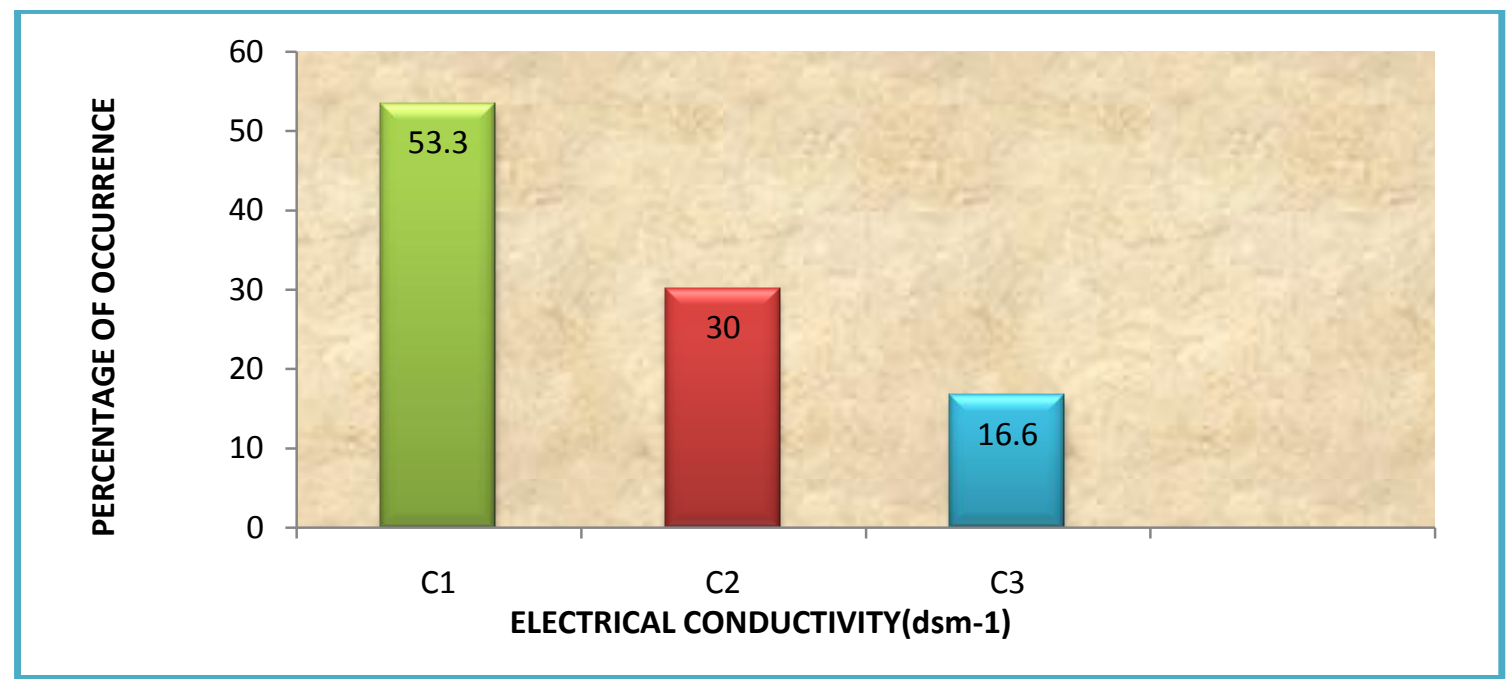

Figure. 5 Residual Sodium Carbonate Rating

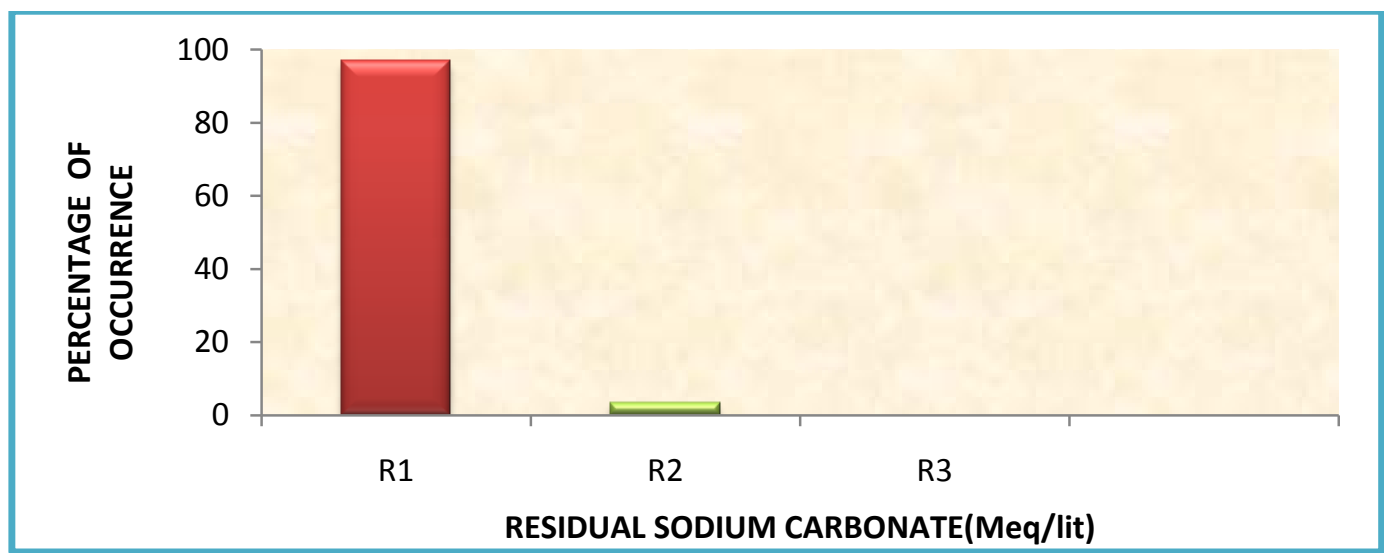

Figure. 6 Sodium Absorption Ratio

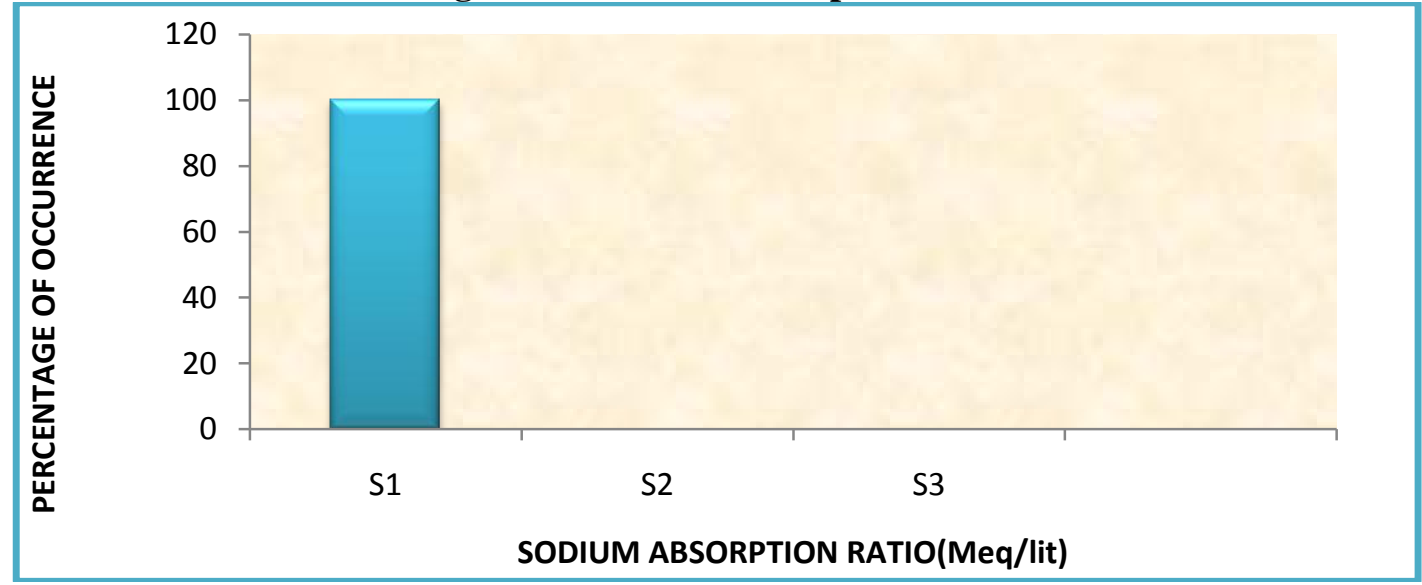

